\title{
Stellar and Circumstellar Activity in the Be Star EW Lac from the Multi-site 1993 Campaign
}

\author{
A.M. Hubert ${ }^{1}$, M. Floquet ${ }^{1}$, R. Hirata ${ }^{2}$, D. McDavid ${ }^{3}$, J. Zorec $^{4}$, D. \\ Gies $^{5}$, M. Hahula ${ }^{5}$, E. Janot-Pacheco ${ }^{6}$, E. Kambe ${ }^{7}$, N.V. Leister ${ }^{6}$, S. \\ Stefl $^{8}$, A. Tarasov ${ }^{9}$ \\ ${ }^{1}$ Dasgal, UMR8633 du CNRS, Observatoire de Paris-Meudon, F-92195 \\ Meudon, France
}

${ }^{2}$ Department of Astronomy, Kyoto University, Kyoto 606-01, Japan

${ }^{3}$ Limber Observatory, Timber Creek Road, P.O. Box 65599

${ }^{4}$ Institut d'Astrophysique de Paris - CNRS, 98bis Bd Arago, 75014-Paris, France

${ }^{5}$ CHARA and Department of Physics and Astronomy, Georgia State University, Atlanta, GA 30303-3083, USA

${ }^{6}$ Instituto Astronomico e Geofisico, Universidade de Sao Paulo, Caixa Postal 3386, 01060-970 Sao Paulo, Brazil

${ }^{7}$ Department of Geoscience, National Defense Academy, Yokosuka, Kanagawa 239, Japan

${ }^{8}$ Astronomical Institute, Academy of Sciences, CZ-251 65 Ondrejov, Czech Republic

${ }^{9}$ Crimean Astrophysical Observatory, Nauchny, Crimea, 334413, Ukraine

\begin{abstract}
The Be shell star EW Lac was observed in September 1993 during a multi-site campaign. Results from visual spectroscopy and polarimetry are summarized here. He I 6678 profiles have been compared to previous observations held in $\mathbf{1 9 8 9}$ and show an additional complex and highly variable circumstellar component which can due to material expelled from the star just prior to these observations. Two groups of frequencies are found again in 1993 observations compared with 1989 ones. In the frame of $n r p$, they could be associated to low degree g-modes.
\end{abstract}

\section{Introduction}

EW Lac (HD 217050, B3 III, V $=5.35, V \sin i=340 \mathrm{~km} / \mathrm{s}$ ) is one of Be stars that have exhibited an alternation of quiescent and active shell phases. It has undergone an active shell phase since 1976. Multiperiodicity has been detected both in light and photospheric line profiles so this star has been thought as a good candidate for a search of relation between short-term line profiles and light 
variations, and shell activity.

As the state of the envelope has been continuously varying during the campaign, one of the essential points was to confirm the multiperiodicity and to identify modes.

\section{The multi-site, multi-wavelength and multi-technique campaign}

Dates: from 1993 August 30 to 1993 September 9

Techniques: Visible spectroscopy, photometry, polarimetry, UV spectroscopy Sites for visible spectroscopy and polarimetry:

He I 6678 line (779 spectra): Haute Provence, Kitt Peak, Dominion, Okayama, Crimea, Ondrejov

$\mathrm{H} \alpha$ line (513 spectra): Kitt Peak, Ondrejov

Polarimetry (54 measurements): McDonald, Crimea.

\section{He I 6678 line}

In 1993 the structure of the line is rather different from that previously observed in the first campaign in 1989 (OHP), an additional variable and complex circumstellar (CS) component is present and the $\mathrm{V}$ and $\mathrm{R}$ outer emission components are stronger.

\subsection{Circumstellar contribution in $\mathbf{1 9 9 3}$}

The mean 1989 profile can be easily fitted by a Gaussian which is taken in the following as a reference profile. Then, to display the circumstellar contribution quite well present in 1993 data, we divided each individual profile by the 1989 Gaussian fit.

The circumstellar contribution can be described as the superimposition of:

- a broad variable absorption for which the strength and the full width are decreasing over the run. Its central position moves slightly and continuously to the short wavelengths from $-25 \mathrm{~km} / \mathrm{s}$ on August 30 to $-60 \mathrm{~km} / \mathrm{s}$ on September 8 . This component, that we call "pseudo-photosphere" is probably due to material ejected prior to the beginning of our run and whose opacity is decreasing as it expands.

- a narrower blue absorption centered around $-200 \mathrm{~km} / \mathrm{s}$, rather stable in position but highly variable in intensity. This feature is hardly seen in the second part of the run. On OHP spectra, which in our sample have the highest S/N ratios, at the level of this absorption several discrete components, not regularly spaced but with $\delta t \leq 0.1 \mathrm{~d}$ and a mean apparent acceleration of about 900 $\mathrm{km} / \mathrm{s} / \mathrm{d}$ are seen at the beginning of the run. They could be explained by sporadic expulsion of material ("puffs").

- two rather strong and narrow components fairly stable in position, at -13 $\mathrm{km} / \mathrm{s}$ (i.e. the stellar radial velocity) and $+50 \mathrm{~km} / \mathrm{s}$, increasing in intensity over the run contrary to the broad "pseudo-photosphere" one. The first one could be the result of mass transfer from the quasi-photosphere to the envelope suggesting 
that this envelope is detached from the stellar disc. The second one could be associated with an infall of a fraction of the ejected material. Both form the core of the complex CS component.

- two weak $V$ and $R$ emission peaks whose velocity separation decreases over the run from 800 to $720 \mathrm{~km} / \mathrm{s}$. The radial velocity of the red peak slightly moves from +380 to $+320 \mathrm{~km} / \mathrm{s}$ as the violet one is roughly stable around $-400 \mathrm{~km} / \mathrm{s}$. Such a behaviour is consistent with a detached ring progressively expanding from the stellar disc.

The additional broad variable "pseudo-photospheric" component and the $\mathrm{V}$ and $\mathrm{R}$ emissions can be an indication of an ejected slab/ring first rotating closely to the star, then progressively detaching and slowly expanding over some days with decreasing opacity. A modelling of the behaviour of the broad "pseudophotospheric" component and the $\mathrm{V}$ and $\mathrm{R}$ emissions, and polarization decrease over the run is in progress.

\subsection{EW, FWHM and RV variations}

All these quantities are highly variable and show a short-term variation superimposed on a mid-term one. Results concerning the search of frequencies are given below.

\section{Ho line}

This line shows a strong double emission peak structure, whose total intensity is smaller in 1993 with respect to 1989 . The intensity of $V$ and $R$ components are variable. In 1993, the V/R ratio is oscillating between 1.02 and 1.16 , and the $\mathrm{V}$ emission shows a monotonic increase on which a short-term variation is superimposed and the $\mathrm{R}$ emission only shows a short-term variability, these two quantities being sometimes in phase and sometimes out of phase.

\section{Polarization}

The polarization obtained simultaneously shows a strong correlation with the circumstellar contribution. It is higher when the equivalent width is stronger i.e. the circumstellar contribution is important, specially when the narrow blue absorption observed on quotients (see above) is maximum.

\section{Evidence of an orbiting circumstellar cloud}

A non-periodic component is seen on residues, slowly crossing the He I line profile on 1993 September 3. This feature can be seen on OHP data, then on DAO and KPNO data. It can be followed during at least 14 hours. Its acceleration ( 620 $\mathrm{km} / \mathrm{s} / \mathrm{d}$ ) is too low to be a corotating stellar spot, so this feature originates in the inner part of circumstellar layers. At the stellar surface, the acceleration is about $1650 \pm 300 \mathrm{~km} / \mathrm{s}$.

To derive rough physical characteristics of the cloud producing the non-periodic sharp absorption in the He I 6678 line, we can think of it as a circular absorbing slab with radius $R_{b}$ crossing the stellar disc. The residual central intensity $r_{0}$ 
produced by the absorption feature is then given by:

$r_{0}=\left(R_{b} / R_{\star}\right)^{2}\left(1-B_{0}\left(T_{r}\right) / F_{\star}\right)\left(1-\exp \left(-\tau_{0}\right)\right)$

where $B_{0}\left(T_{r}\right) / F_{\star} \sim 0.41 \pm 0.15$ for excitation temperatures $0.810^{4}<T_{r}<$ $1.210^{4} \mathrm{~K}$ typical for environments producing shell spectra. Assuming a marginal value $\tau_{0} \sim 1$, and the measured value $r_{o}=0.009 \pm 0.001$ we derive: $R_{b} / R_{\star}=$ $0.16 \pm 0.02 ; 4.510^{-13}<\Delta M / M_{\odot}<2.310^{-11}$ and $0.4 R_{\star}<R-R_{\star}<0.8 R_{\star}$.

\section{Time series analysis}

Main typical quantities of $\mathrm{He} \mathrm{I} 6678$ and $\mathrm{H} \alpha$ were investigated, such as equivalent width (EW), full width at half maximum (FWHM), centroid velocity (RV) of the absorption component of He I 6678, intensity and equivalent width of variable $\mathrm{V}$ and $\mathrm{R}$ emissions of the $\mathrm{He} \mathrm{I}$ line, and intensity of $\mathrm{V}$ and $\mathrm{R}$ components of $\mathrm{H} \alpha$ emission line. He I full line profile variability $(l p v)$ analysis was then performed. Investigation of multi-periodicity in main quoted variable quantities and in $l p v$ was done using the same methods as in Hubert et al. (1997). A comparison was made between results deduced from analyses of 1989 and 1993 data.

Results of time-series analysis allow to us to assert the presence, in 1989 and 1993 , of two groups of frequencies having a stellar origin: $1.55,3.20 \mathrm{c} / \mathrm{d}$ and $1.39,2.77 \mathrm{c} / \mathrm{d}$. Both can be considered as fundamental and first harmonic of non-sinusoidal signals. Frequencies $1.39,1.55 / 1.60$ and $1.22 \mathrm{c} / \mathrm{d}$ are distinct from the rotational frequency (mean estimation $0.77 \pm 0.18 \mathrm{c} / \mathrm{d}$ ), but it cannot be clearly determined if one among these values is a submultiple.

If the assertion of prograde modes corresponding to 1.39 and $1.55 / 1.60 \mathrm{c} / \mathrm{d}$ is correct, it will allow to put an upper limit on the rotational frequency $(<0.70$ $\mathrm{c} / \mathrm{d})$. The $1.60 \mathrm{c} / \mathrm{d}$ frequency is the more important one detected in 1989 in the He I 6678 line profile. In terms of $n r p$ it could be associated with a low degree prograde sectorial g mode $\ell=-m=2$. In 1993 it changes progressively from $1.60 \mathrm{c} / \mathrm{d}$ in the wings towards $1.50 \mathrm{c} / \mathrm{d}$ in the core indicating some damping effect in upper photosphere, this line being known to be very sensitive to non-LTE effect (Auer and Mihalas 1973). Other frequencies 0.91 and $1.22 \mathrm{c} / \mathrm{d}$, dominating 1993 data, are mainly present in the blue wing of the line. They cannot be associated with $n r p$. The $1.22 \mathrm{c} / \mathrm{d}$ frequency could be a beat frequency between 2.76 and $1.55 \mathrm{c} / \mathrm{d}$ or $/$ and a multiple $(\times 2)$ of the rotational frequency. A $1.25 \mathrm{c} / \mathrm{d}$ frequency is weakly present in 1989 data and appears among frequencies (1.28 c/d) derived by Pavlovski et al. (1997), and by Percy (private communication) from the August/September 1993 photometric campaign. Both frequencies 0.93 and $1.22 \mathrm{c} / \mathrm{d}$ are found in short-term variations of main quantities and $l p v$ of He I 6678; they are probably linked to outflows modulated by rotation or with a coupling between frequencies presumedly associated with $n r p$ and rotation.

\section{References}

Auer, L.H., Mihalas, D. 1973, ApJS 25, 433

Hubert, A.M., Floquet, M., Hao, J.X. et al. 1997, A\&A 324, 929

Pavlovski, K., Harmanec, P., Božić, H. et al. 1997, A\&AS 125, 75 